(c) Journal of Applied Mathematics \& Decision Sciences, 4(2), 125-141 (2000)

Reprints Available directly from the Editor. Printed in New Zealand.

\title{
Mean Action Time for Diffusive Processes
}

\author{
KERRY LANDMAN \\ k.landman@ms.unimelb.edu.au \\ Department of Mathematics and Statistics, University of Melbourne, Parkville, Victoria, Aus- \\ tralia \\ MARK MCGUINNESS \\ mark.mcguinness@vuw.ac.nz \\ School of Mathematical and Computing Sciences, Victoria University of Wellington, Wellington, \\ New Zealand
}

\begin{abstract}
For a number of diffusive processes involving heat and mass transfer, a convenient and easy way to solve for penetration time or depth is to consider an averaged quantity called mean action time. This approach was originally developed by Alex McNabb, in collaboration with other researchers. It is possible to solve for mean action time without actually solving the full diffusion problem, which may be nonlinear, and may have internal moving boundaries. Mean action time satisfies a linear Poisson equation, and only works for finite problems. We review some nice properties of mean action time, and discuss some recent novel applications.
\end{abstract}

Keywords: Mean Action Time, Diffusion, Time, Applications

\section{Introduction}

Diffusive processes occur in many contexts, including heat and mass transfer. Certainly a common question is how long does a process take? This question cannot be answered simply, because diffusive processes take an infinite amount of time to come to equilibrium. Therefore other measures of process time need to be defined. For example in a heat transfer process, we could consider a fixed position $\mathbf{r}$ and determine the time taken for the temperature there to reach some fixed fraction of the final equilibrium value. Alternatively, one could take a more averaged approach and determine when the average temperature is a fixed fraction of final equilibrium value.

An attractive alternative is to use mean action time. The mean action time is a measure of the time taken for a disturbance introduced at the boundary to reach an observation point, and hence is a function of position. It is especially useful if the process is such that a steep front moves through the body, in which case it measures the time at which the front passes through position $\mathbf{r}$. 
The concept of mean action time has been fully developed by McNabb [6], [7], [9], [8], [11] to quantify behaviour in non-linear diffusion problems in various geometries. Here we will discuss the definition, and review some of the properties of the mean action time. Recently, the mean action time has been used successfully to shed light on some novel applications, which will be discussed here.

\section{Linear and Nonlinear Diffusion Problems}

We begin by considering a linear diffusion problem with a constant diffusivity $D$ - this could represent a heat conduction problem or a mass transfer problem. We let $c$ denote the conserved quantity, and then the flux is given as $-D \nabla c$. At this stage we will consider an Eulerian co-ordinate scheme, and we do not specify the number of spatial variables.

$$
\frac{\partial c}{\partial t}=D \nabla^{2} c
$$

We suppose that the system is initially uniform with $c(\mathbf{r}, t)=c_{0}$, of finite extent. The value of $c$ at an outer boundary is changed at time zero, and the system undergoes changes in $c$, tending towards some final uniform equilibrium value $c_{\infty}$.

The mean action time is a measure of the time taken for a disturbance introduced at the boundary to diffuse past any observation point and hence is a function of position denoted by $T(\mathbf{r})$. It is defined in such a way that it is located in the neighbourhood of extrema in $\partial c(\mathbf{r}, t) / \partial t$, as illustrated in Figure (1).

As in [9], we define the mean-action time for the linear problem to be

$$
T(\mathbf{r})=\frac{\int_{0}^{\infty} t \frac{\partial c}{\partial t} d t}{\int_{0}^{\infty} \frac{\partial c}{\partial t} d t},
$$

where the time derivative term in the integrand typically plays the role of a peaked function which serves to concentrate $t$ in the neighbourhood of extrema of $\partial c(\mathbf{r}) / \partial t$. When a steep front is present this formula acts to select the time $T(\mathbf{r})$ as the time of arrival of the front.

For the nonlinear problem, the diffusivity is a function of $c$, written as $D(c)$, and the diffusion equation takes the form

$$
\frac{\partial c}{\partial t}=\nabla \cdot(D(c) \nabla c)
$$




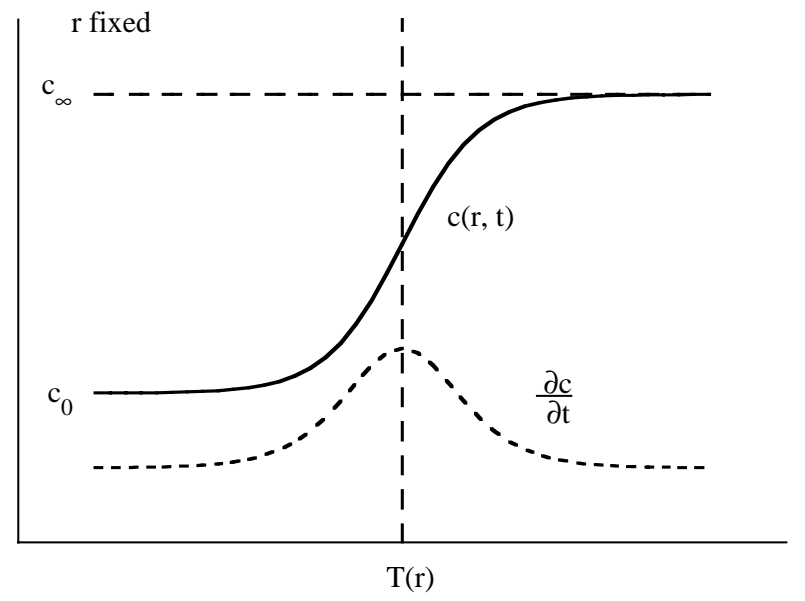

Figure 1. A sketch of concentration and its partial time derivative versus time.

The definition of mean-action time for the nonlinear problem can be written as

$$
T(\mathbf{r})=\frac{\int_{0}^{\infty} t \frac{\partial}{\partial t}\left(\int_{c_{0}}^{c(\mathbf{r}, t)} D(c) d c\right) d t}{\int_{0}^{\infty} \frac{\partial}{\partial t}\left(\int_{c_{0}}^{c(\mathbf{r}, t)} D(c) d c\right) d t} .
$$

It is clear that setting $D$ to be a constant in (4) yields the definition for the linear diffusion problem (2). Hence the definitions for the nonlinear case are a generalisation of the linear case.

For some mass transfer problems, for example in soil mechanics ([14], [15]), it is more convenient to use Lagrangian co-ordinates. In this case the physical coordinate may measure the total amount of the conserved quantity from $\mathbf{r}=0$ to some nonzero $\mathbf{r}$. Furthermore, some moving boundary problems can be reformulated into a constant domain diffusion problem. For these cases, the mean action time can also be defined as above. See section 3 for an application of this.

Internal moving boundaries associated with freezing and thawing problems are also accommodated with the same nonlinear definition of mean action time [9]. 


\subsection{Simpler Form for $T$ and Geometric Interpretation}

Let us first consider the case of constant $D$, and $T$ defined by (2). We integrate by parts, being careful to avoid any divisions by zero.

$$
\begin{aligned}
T(\mathbf{r}) & =\frac{\int_{0}^{\infty} t \frac{\partial\left(c-c_{\infty}\right)}{\partial t} d t}{\int_{0}^{\infty} \frac{\partial c}{\partial t} d t} \\
& =\frac{1}{\left(c_{\infty}-c_{0}\right)} \int_{0}^{\infty}\left(c_{\infty}-c\right) d t .
\end{aligned}
$$

Hence,

$$
\left(c_{\infty}-c_{0}\right) T=\int_{0}^{\infty}\left(c_{\infty}-c\right) d t .
$$

This can be interpreted geometrically using Figure (2). There we consider the $c$ profile as a function of $t$ for a fixed position $\mathbf{r}$. The areas shaded on either side of the mean action time are equal using (7), showing how it corresponds to locating a front in shock theory.

For the nonlinear problem it is convenient to introduce the Kirchoff transformation [2]

$$
V(c)=\int_{c_{0}}^{c(\mathbf{r}, t)} D(c) d c .
$$

Then considering (4), we can integrate by parts, again avoiding any infinities.

$$
\begin{aligned}
T(\mathbf{r}) & =\frac{\int_{0}^{\infty} t \frac{\partial V}{\partial t} d t}{\int_{0}^{\infty} \frac{\partial V}{\partial t} d t} \\
& =\frac{\int_{0}^{\infty} t \frac{\partial\left(V-V\left(c_{\infty}\right)\right)}{\partial t} d t}{\int_{0}^{\infty} \frac{\partial V}{\partial t} d t} \\
& =\frac{1}{V\left(c_{\infty}\right)} \int_{0}^{\infty}\left(V\left(c_{\infty}\right)-V\right) d t .
\end{aligned}
$$

Hence,

$$
V\left(c_{\infty}\right) T=\int_{0}^{\infty}\left(V\left(c_{\infty}\right)-V\right) d t
$$




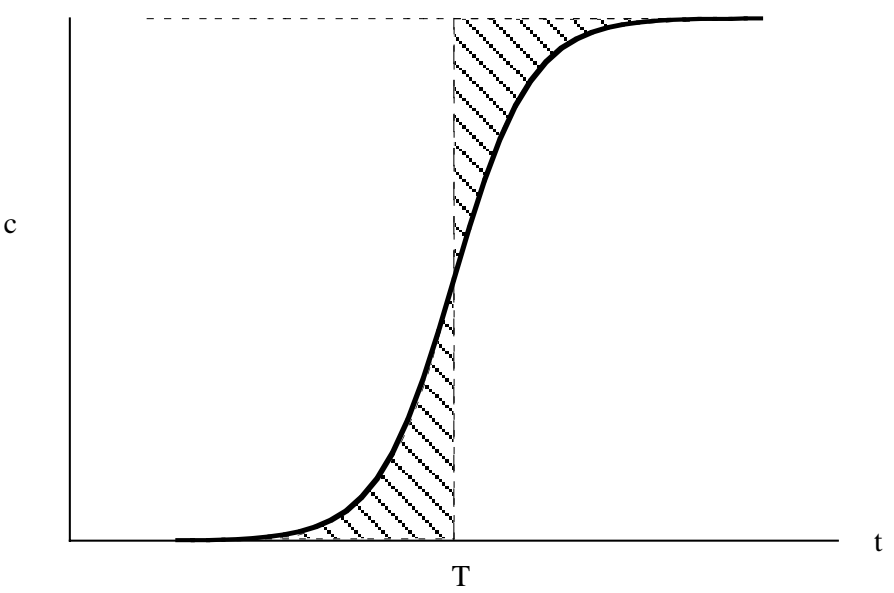

Figure 2. A sketch illustrating the location of the mean action time $T$ in a typical concentration versus time plot, at some fixed value of $\mathbf{r}$, with the two shaded areas being equal in value.

Noting that $V\left(c_{0}\right)=0$, this has the same geometric interpretation as in the linear case for equation (7), if $c$ is replaced by $V(c)$. Hence in the nonlinear generalisation of mean action time, there is the geometric interpretation that the mean action time is placed in such a way as to give equal areas, in a plot of $V(c)$ against time at any point.

\subsection{Poisson Equation for $T(\mathrm{r})$}

For the linear problem, we can differentiate the simplification (6) for mean action time, and use (1) to give

$$
\nabla^{2} T(\mathbf{r})=-\frac{1}{\left(c_{\infty}-c_{0}\right)} \int_{0}^{\infty} \nabla^{2} c d t=-\frac{1}{\left(c_{\infty}-c_{0}\right)} \frac{1}{D} \int_{0}^{\infty} \frac{\partial c}{\partial t} d t=-\frac{1}{D} .
$$

Hence, $T$ satisfies a Poisson equation. The boundary conditions for $T$ can be obtained from the boundary conditions for $c$. This is relatively straightforward for problems on bounded domains, and may also be used for some unbounded problems. 
To obtain an analogous expression for the nonlinear problem, we first rewrite the partial differential equation (3) as

$$
\frac{\partial c}{\partial t}=\nabla^{2} V .
$$

Then, as before, we differentiate the mean action time expression (11), using (14), to obtain

$$
\begin{aligned}
\nabla^{2} T(\mathbf{r}) & =-\frac{1}{V\left(c_{\infty}\right)} \int_{0}^{\infty} \nabla^{2} V \\
& =-\frac{1}{V\left(c_{\infty}\right)} \int_{0}^{\infty} \frac{\partial c}{\partial t} d t \\
& =-\frac{\left(c_{\infty}-c_{0}\right)}{\int_{c_{0}}^{c_{\infty}} D(c) d c} .
\end{aligned}
$$

Again this is an appropriate generalisation of the linear problem. More importantly, we see that two nonlinear systems with the same boundary and initial conditions have identical mean action time if the quantity

$$
\int_{c_{0}}^{c_{\infty}} D(c) d c
$$

is the same in both cases. This has been used to good effect in a filtration problem discussed below.

Moving internal boundaries associated for example with freezing and thawing problems are also amenable to this approach. Latent heat effects at the internal boundary are accommodated by considering internal energy in the time derivative term, which leads to a change in internal energy term on the right-hand side of the Poisson equation, a generalisation of equation (17). See [9] for more details.

\section{Application to Filtration}

The modelling of pressure filtration of flocculated suspensions [4] using compressional rheology and a knowledge of compressional yield stress and hydraulic resistance leads to nonlinear diffusion-convection equations in the volume fraction of solids $(\phi)$, within a one-dimensional domain. This domain reduces in size as time increases, and represents the distance between the fixed membrane which allows water to filter through and the piston position, where a constant pressure is ap-

plied. Using a Lagrangian approach, this problem is recast as a standard non-linear diffusion equation

$$
\frac{\partial e}{\partial t}=\frac{\partial}{\partial w}\left[\Delta(e) \frac{\partial e}{\partial w}\right],
$$


where the void ratio $e=(1-\phi) / \phi$ is a function of the material co-ordinate $w$ and time $t$, and $0<w<1$. The boundary conditions are $e(0, t)=e_{\infty}$ and $\frac{\partial e}{\partial w}(1, t)=0$, and the initial condition is $e(w, 0)=w_{0}$. The diffusivity $\Delta(e)$ is illustrated in figure (3).

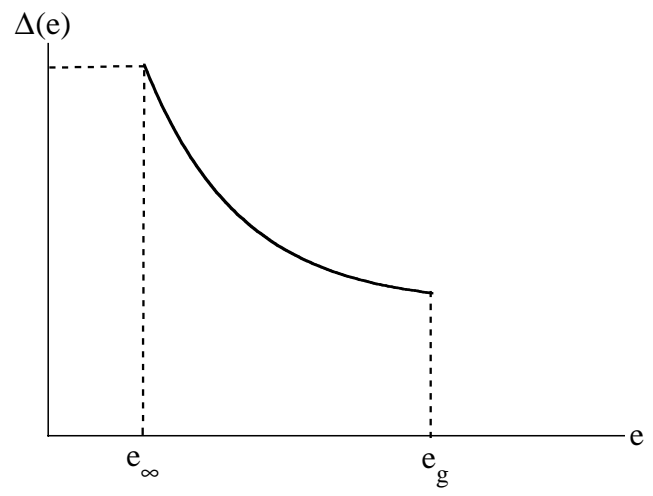

Figure 3. A sketch of diffusivity as a function of void ratio, for the pressure filtration problem.

In this process, it is desirable to estimate the dimensionless filtration time $T_{f}$, here defined to be the time when the average solids fraction is $95 \%$ of the equilibrium value (maximum possible value) $\phi_{\infty}$. The nonlinear problem can be numerically solved for the profile $e$ and hence $\phi$, but a more practical engineering estimate of the filtration time is required. The aim is to find a simpler non-linear diffusion problem, which can be solved analytically to find expressions for $T_{f}$.

The choice of filtration time $T_{f}$ for our non-linear system is largely arbitrary, and a choice that is more amenable to analysis is that of mean action time $T(w)$. Such a choice also has the attraction of providing insight into the behaviour of the fitration system. We replace our non-linear system with another system (a system with a different $\Delta(e)$ ) that is more amenable to solution, and we ensure that the two systems have identical mean action time by requiring that, in the replacement, the quantity

$$
\int_{e_{\infty}}^{e_{0}} \Delta(e) d e
$$

is preserved (using an analagous result to (18) for $\Delta$ ).

The use of mean action time as a measure of how long it takes for a diffusion process to proceed to practical completion is discussed in previous sections, and will be seen 
here to give results that match very closely the filtration times $T_{f}$ obtained from numerical solutions.

We wish to replace our non-linear diffusion problem by another diffusion problem that can be solved analytically. This leads us to seek (piecewise) linear replacements for $\Delta(e)$, with the desirable properties that $\Delta(e)=0$ for $e>e_{g}$, and that $\Delta(e) \rightarrow$ $\Delta\left(e_{\infty}\right)$. One such choice gave excellent comparisons with the solutions of the real system, namely

$$
\Delta_{\mathrm{eff}}=\Delta\left(e_{\infty}\right) H\left(e^{*}-e\right),
$$

where $H$ is the unit step function. Equality of mean action times is assured by requiring that

$$
e^{*}=e_{\infty}+\int_{e_{\infty}}^{e_{0}} \Delta(e) d e
$$

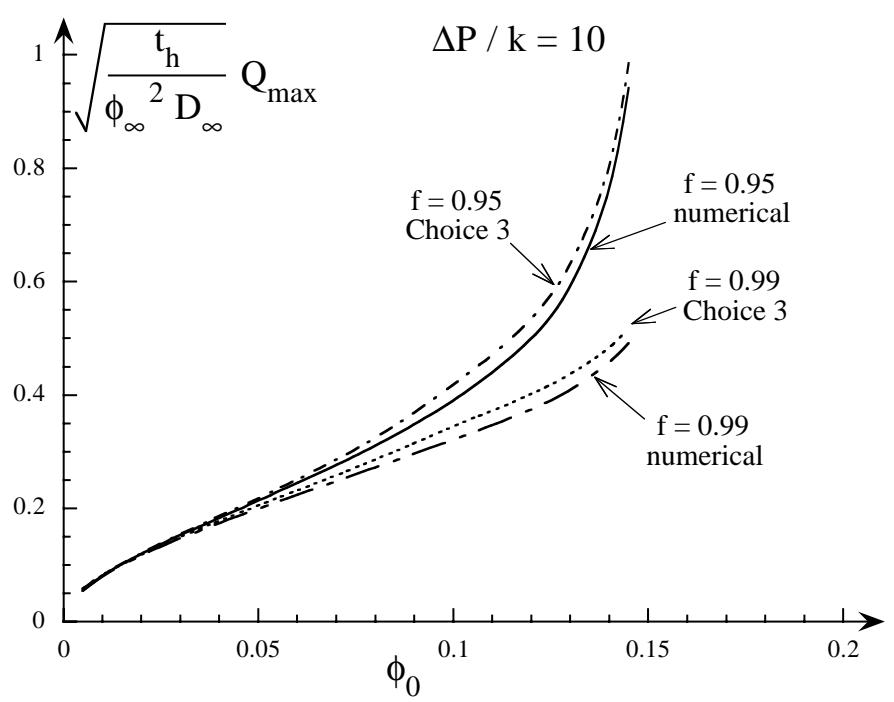

Figure 4. A comparison of the numerical and approximate analytical solutions for throughput versus initial solid volume fraction, for the filtration problem. Here $t_{h}$ is the constant handling time, $D_{\infty}=D\left(\phi_{\infty}\right)$ and $\Delta P / k$ is the fixed applied pressure. Choice 3 was the best of the three choices discussed in [4].

For this choice of $\Delta_{\text {eff }}$, the linear diffusion problem can be solved exactly. At early times there is a similarity solution with an internal moving boundary $w_{c}(t)$. The similarity solution is valid until such time as $w_{c}(t)=1$. For longer times, the diffusivity is a constant and the problem may be solved by separation of variables. The Fourier coefficients are found by matching to the similarity solution, and an explicit expression for the filtration time can be determined [4]. 
See figure (4) for a comparison of numerical solutions and the approximate analytic solutions obtained using $\Delta_{\text {eff }}$. Plotted on the vertical axis is dimensionless maximum throughput, the total amount of solid processed in a batch operation, and this quantity is inversely proportional to $T_{f}^{1 / 2}$. On the horizontal axis is the initial volume fraction of solids. The factor $f$ is the fraction of the equilibrium solids fraction at which the process is interrupted.

\section{Application to Water Transport in Human Eye Lenses}

Magnetic resonance microscopy (MRM) has been used to study the kinetics of water transport in human eye lenses [13]. NMR signal intensity from the lenses decreases with time corresponding to a decrease in concentration of $\mathrm{H}_{2} \mathrm{O}$ and replacement by $\mathrm{D}_{2} \mathrm{O}$ within the lenses. A statistically significant correlation has been found between the rate of NMR signal loss from the lens nuclei and the age of the lenses.

The results show that as lenses age there is a reduction in the rate at which water can enter the cells of the lens nucleus via the epithelium and cortex. A decrease in the rate of transport of water, nutrients and anti-oxidant species would be expected to lead to increased damage to lenses with age, and is a potential cause of presbyopia and senile cataract.

In order to establish a theoretical basis for analysis of the changes in water proton signal observed in the MR images, a simple model for the exchange process was developed. In the following analysis, the exchange of $\mathrm{H}_{2} \mathrm{O}$ in the lens nucleus with $\mathrm{D}_{2} \mathrm{O}$ in the surrounding artificial aqueous humour (AAH) is assumed to be governed by an effective diffusion coefficient $D$, which itself is assumed to be isotropic and uniform throughout the lens. As well as establishing appropriate equations for fitting the experimental data, this modeling enables the characteristic parameters to be objectively defined, facilitating a correlation of the exchange process with age.

For three-dimensional modelling of the $\mathrm{D}_{2} \mathrm{O} / \mathrm{H}_{2} \mathrm{O}$ exchange in an ellipsoidal shaped lens, modelled by a diffusion equation with a step change in the (initially constant everywhere) concentration at the surface of the lens, it is possible to calculate an analytical solution using separation of variables. However, the expressions are complex and clumsy and it is convenient to use the mean action time to come to some understanding of appropriate dimensional quantities.

We approximate the lens as an ellipsoid,

$$
\frac{x^{2}}{L^{2}}+\frac{y^{2}}{M^{2}}+\frac{z^{2}}{N^{2}}=1
$$

where $2 L, 2 M$ and $2 N$ are the lengths of the principal axes. The mean action time satisfies the Poisson equation (13) inside the ellipsoid, with $T=0$ everywhere on 
the surface of the ellipsoid since the concentration of $\mathrm{H}_{2} \mathrm{O}$ there is $c_{\infty}$. The solution in a three dimensional ellipsoid is

$$
T(x, y, z)=\frac{1}{2 D\left(\frac{1}{L^{2}}+\frac{1}{M^{2}}+\frac{1}{N^{2}}\right)}\left[1-\left(\frac{x^{2}}{L^{2}}+\frac{y^{2}}{M^{2}}+\frac{z^{2}}{N^{2}}\right)\right] .
$$

The largest mean action time, at the centre of the lens, is given by

$$
T(0,0,0)=\frac{1}{2 D\left(\frac{1}{L^{2}}+\frac{1}{M^{2}}+\frac{1}{N^{2}}\right)} .
$$

Solving the original diffusion problem using separation of variables gives a solution which decays exponentially in time. The slowest decay rate in this solution is related to the mean action time. A generalised relationship between the decay constant $b$ and the mean action time at the lens nucleus takes the form

$$
b=\pi^{2} /(8 T(0,0,0))=\frac{\pi^{2} D}{4}\left(\frac{1}{L^{2}}+\frac{1}{M^{2}}+\frac{1}{N^{2}}\right)
$$

This equation shows how the decay rate depends on the size and shape of the lens as well as on the diffusivity of water in the lens. Since lens size (determined by the values of $L, M, N$ ) certainly changes with age, a procedure is required for deciding whether observed changes in decay rates are due to changes in lens size or to changes in lens shape.

Equation (25) gives a method for correcting the observed signal decay rates for the effects of differences in lens size. From (25), a plot of $b /\left(\frac{1}{L^{2}}+\frac{1}{M^{2}}+\frac{1}{N^{2}}\right)$ should be independent of lens size - any significant trends in this data will be due to changes in lens morphology with age, and expressible as an effective diffusivity.

By simple mathematical modeling of the experimental results it was concluded that the decrease in transport rates was due to two factors, a continuous increase in lens size with age, and a change in lens morphology causing a decrease in apparent diffusivity of water (and other low molecular weight species) either throughout the lens nucleus or within in a region surrounding the nucleus. The results support the hypothesis that such factors may contribute to a continual decrease in lens function with age, resulting in the onset of presbyopia and in some cases, cataract formation.

\section{Application to Water Uptake in a Cereal Grain}

In trying to understand how to optimise the cooking of whole grains for the manufacture of breakfast cereals, it is recognised that water and heat must enter the 
grain. Heat penetration is a relatively fast and simple diffusion process. Water uptake by the whole grain can be modelled by a nonlinear diffusion equation, with a steep wetting front penetrating the grain. Here we will show how the mean action time has shed light on the tracking of the moisture front with time.

Moisture movement through whole corn kernels is considered in the paper by Syarief et al [17] and for whole wheat grains by Stapley [16]. The moisture diffusivity is determined to be strongly dependent on moisture content and can be fitted with either a power law or an exponential function. We consider the exponential form here.

Once the equations have been made dimensionless, we obtain (see [5]) the diffusion equation for the scaled moisture content $m$,

$$
\frac{\partial m}{\partial \tau}=\nabla \cdot\left(e^{\beta m} \nabla m\right) .
$$

Here the time $\tau$ is scaled by a representative length scale and by the diffusivity, evaluated at the equilibrium value of the moisture content, corresponding to a wet grain. The initial condition is $m=-1$ everywhere inside the grain (relatively dry conditions) and the boundary condition at the grain surface is $m=0$ (relatively wet) for $\tau>0$. The parameter $\beta$ typically takes values about 4 ([17]).

Substituting the exponential form for the diffusivity into the simplified form (11) for the mean action time gives

$$
T(\mathbf{r})=\frac{\int_{0}^{\infty}\left(1-e^{\beta m}\right) d \tau}{1-e^{-\beta}} .
$$

We will now consider the maximum mean action time, that is, the time to wet the centre of the grain. This is

$$
T(0)=\frac{1}{D_{w e t}}\left[\frac{\beta}{1-e^{-\beta}}\right]\left[\frac{1}{2\left(\frac{1}{L^{2}}+\frac{1}{M^{2}}+\frac{1}{N^{2}}\right)}\right] .
$$

A significant way to rewrite the maximum mean action time in dimensional terms is as

$$
T(0)=\frac{1}{D_{\text {equiv }}}\left[\frac{1}{2\left(\frac{1}{L^{2}}+\frac{1}{M^{2}}+\frac{1}{N^{2}}\right)}\right] .
$$

where the equivalent diffusivity is the $\log$ mean of the extreme values taken by the nonlinear diffusivity,

$$
D_{\text {equiv }} \equiv \frac{D_{\text {wet }}-D_{\text {dry }}}{\ln \left(D_{\text {wet }} / D_{\text {dry }}\right)}
$$


where $D_{\text {dry }}$ is the diffusivity of dry grain with $m=-1$ and $D_{\text {wet }}$ is the diffusivity of wet grain with $m=0$.

Even though moisture penetration is a nonlinear diffusion problem, the definition used for mean action time justifies the treatment of the diffusion of moisture as an equivalent linear diffusion problem, at least in terms of the times taken to wet grains. That is, equation (29) is the same as the time to wet that would be obtained from a linear diffusion equation with a diffusion equal to $D_{\text {equiv }}$.

Numerical solutions to equation (26), obtained using Fastflo [1], are plotted in figure (5) together with vertical lines indicating the location of a front associated with the mean action time. Note that this plot shows moisture content versus radius, not versus time. A plot showing moisture content versus time is in figure (6), for three different radii, together with vertical lines indicating the mean action times for those radii. The numerical solutions were for cylindrical geometry, and mean action time is obtained for this geometry from the ellipsoidal case by taking say $N \rightarrow \infty, L=M$, and $r^{2}=x^{2}+y^{2}$. Note that due to the long tails on the moisture content versus time the mean action time is placed a surprisingly long distance (or time) behind the first rise of the moisture content. However, the placement of the mean action time does appear reasonable, on the basis of making the appropriate areas equal.

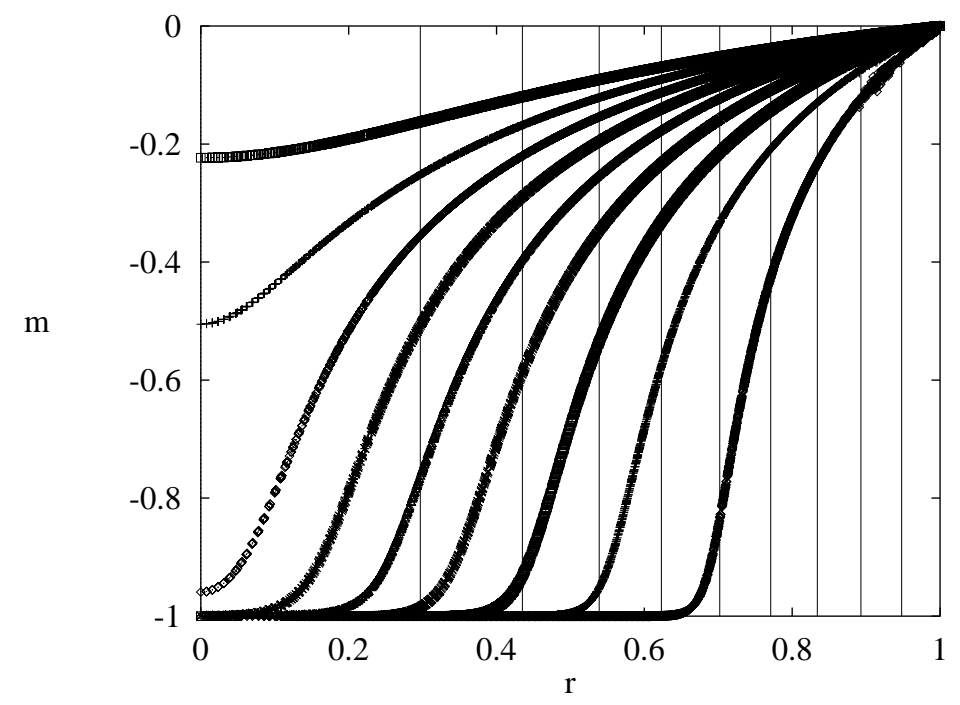

Figure 5. A plot of numerical solutions for moisture content versus radius, in cylindrical geometry, at times $0.1,0.2, \ldots 0.9$ from right to left (moisture is penetrating from $r=1$ to $r=0$ ). Vertical lines show the radii which correspond to mean action times that are equal to the numerical solution times. 


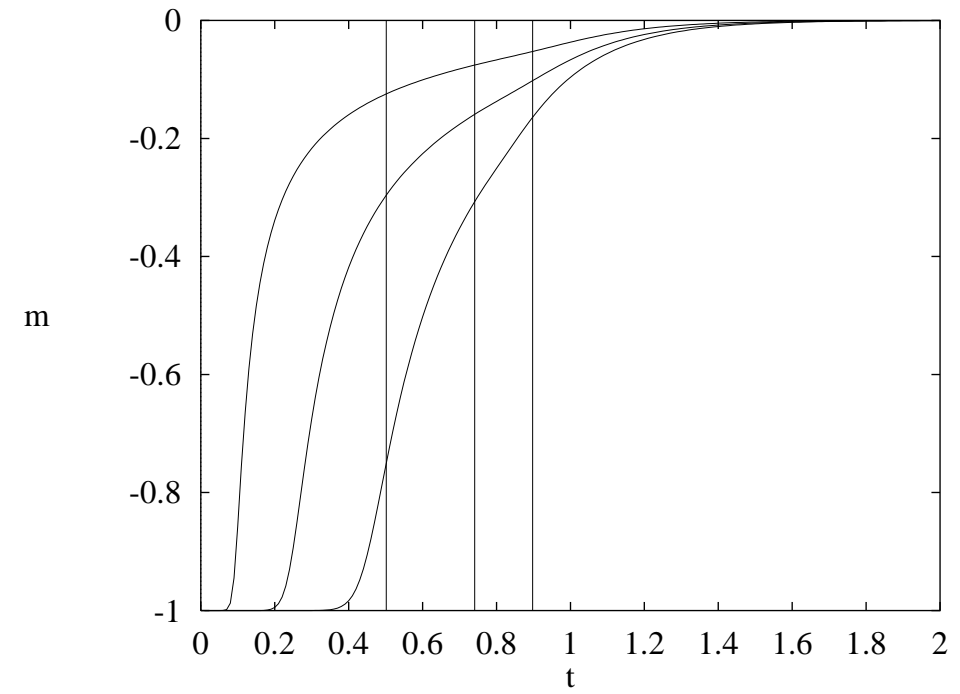

Figure 6. A plot of numerical solutions for moisture content versus time, in cylindrical geometry, at radii $0.7,0.5$ and 0.3 from left to right. Vertical lines show the mean action times for these three radii, in the same order.

It is simple to adapt this work to a spherical grain of radius $R$, since then the solution to the Poisson equation $\nabla^{2} \Phi=-1$ is just $\Phi=\left(R^{2}-r^{2}\right) / 6$. Such a geometry may be more appropriate when dealing for example with milled grains.

\subsection{Comparison with Asymptotic Solutions}

An approximate solution of this nonlinear diffusion problem has been obtained by assuming steady-state diffusion between the outer surface of the grain and a steep wetting front that penetrates the grain [5]. This solution gives a wetting front that moves as illustrated by the dashed line in figure (7). For comparison purposes, the location of the front implied by the mean action time is also shown.

Of interest in figure (7) is the rapid initial penetration of the wetting front according to the asymptotic analysis, compared with the much slower initial penetration predicted by the mean action time analysis. The wetting front for the asymptotic solution is placed where the moisture content first rises from its initial value, whereas the mean action time solution is an exact one, but gives a front that is a significant distance behind the first rise in moisture content. 


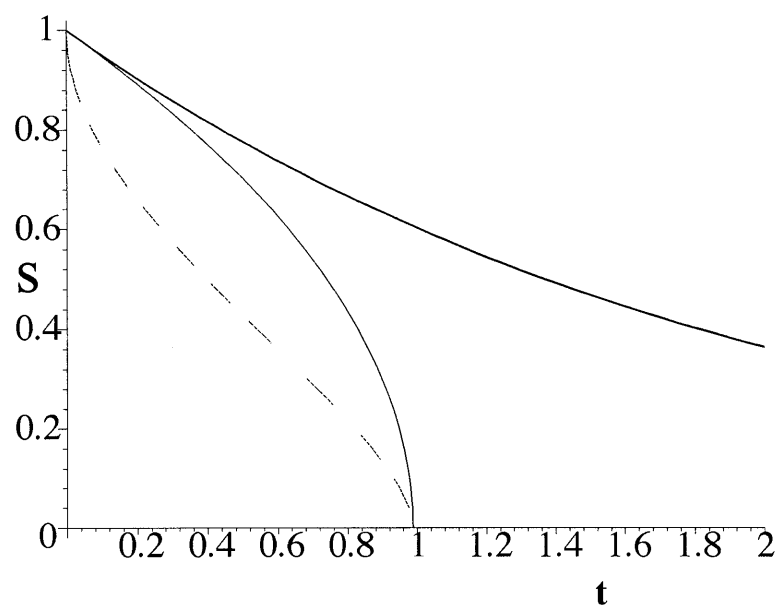

Figure 7. Wetting front locations for grain cooking, from the asymptotic solution (dashed line) and from the mean action time (the middle, solid line). The uppermost and thickest solid line is the front $S(t)$ obtained by substituting the asymptotic solution into the mean action time definition.

To help reconcile these two results, it is possible to substitute the asymptotic solution directly into the defining formula for mean action time, to see if the differences are due in any part to any inadequacies in the asymptotic approximation. We use cylindrical geometry with a cylinder radius equal to one, to simplify the formulae. The mean action time obtained by solving exactly places a sharp front at $s(t)$, where

$$
s^{2}=1-\frac{4 t\left(1-e^{-\beta}\right)}{\beta},
$$

whereas if the asymptotic solution is used to calculate the mean action time, the following formula for wetting front location $S(t)$ is obtained

$$
S^{2}=\exp \left[\frac{-4 t\left(1-e^{-\beta}\right)}{\beta}\right] .
$$

The functions $s(t)$ and $S(t)$ are plotted as solid lines in figure (7). $S$ is the uppermost and thickest solid line. Clearly, from the form of equations 31 and 32, noting the usual expansion for the exponential function, $S(t)$ matches $s(t)$ for small $t$, and deviates at larger $t$ values, as illustrated in figure (7).

It is reassuring that $s$ and $S$ match at small times. At larger times, the asymptotic solution deviates from the exact solution, falling behind due to the assumption in the asymptotics that no diffusion takes place in front of the wetting-front. This 
is also consistent with numerical solutions, which also progress faster than the asymptotic solutions.

Hence we note that the location of the penetrating moisture front is (in the mean action time approach) somewhat behind the initial steep rise in moisture content, due to the long tails. The asymptotic solutions lead to apparently faster initial penetration rates, because they track this first steep rise in moisture content. The asymptotic solutions deviate at later times from both the mean action time and the numerical results, due to an assumption in the asymptotics that the diffusivity in front of the steep moisture shock is zero. Mean action time is a good way to measure and solve for the penetration of moisture into cereal grains.

\subsection{Surface Resistance}

As discussed above, if the surface of the grain is at the external saturation level, then $m=0$ and hence $T=0$ on the grain surface. Then it is easy to solve for $T(\mathbf{r})$ in simple geometries (sphere, ellipsoid, cylinder etc). The largest value of $T$ gives the time for moisture to hydrate the entire grain.

However, for the mass transfer boundary condition with a surface resistance $\lambda$, the analysis is more difficult and only lower and upper bounds on the mean action time can be found ([10], [3]). Depending on the values of $\beta$ and $\lambda$, these bounds may only be useful if the bounds are relatively tight.

\section{Conclusions}

Mean action time is a very useful approach if the details of a diffusive solution are not required, or if the diffusion problem is so complicated or nonlinear that numerical and analytical solutions are problematic. Some may consider the use of mean action time somewhat cavalier in integrating out much of the detail of a solution. However, diffusion problems never quite stabilise, taking infinite time to reach equilibrium, so that the alternative approaches to mean action time also involve making decisions about how much is enough - $90 \%$ of the final concentration, or $95 \%$, or $99 \%$ ? These decisions are rather more arbitrary than the idea of mean action time, which has a nice geometric rationale, placing a penetration distance or an action time at a place that corresponds to locating a shock front to give equal areas on an appropriate time plot.

Mean action time is also remarkable in the range of problems it may be applied to, and in the potential it has to simplify complicated problems, making it the method 
of choice for a range of nonlinear or moving internal boundary problems, freezing and thawing problems [12], and for embedded problems like double-porosity models in geothermal reservoir modelling or pasta drying or iron pellet reduction [10].

\section{Acknowledgments}

The authors wish to thank Alex McNabb for many interesting discussions about mean action time.

\section{References}

1. Fastflo is a finite element package for solving partial differential equations, developed by scientists at the CSIRO Division of Mathematics and Statistics, and marketed by the Numerical Algorithms Group. For further information about Fastflo see: http://www.nag.co.uk/doc/Network/issue15/issue15a.html or http://www.mel.dms.csiro.au/Fastflo/

2. G. Kirchoff. Vorlesungen über die Theorie der Warme. Barth, Leipzig. 1894.

3. K.A. Landman and C. Please. Modelling moisture uptake in a cereal grain. IMA Journal of Mathematics Applied in Business and Industry, 10:265-287, 1999.

4. K.A. Landman and L.R. White. Predicting Filtration Time and Maximising Throughput in a Pressure Filter. AIChE Journal, 43:3147-3160, 1997.

5. P. McGowan and M. McGuinness. Modelling the cooking process of a single cereal grain. Proc of the Mathematics-in-Industry Study Group, (Editor John Hewitt) University of Melbourne, 114-140, 1996.

6. A. McNabb. Asymptotic Behaviour of Solutions of Diffusion Equations. J. Mathematical Analysis and Applications, 51:219-222, 1975.

7. A. McNabb. Asymptotic Behaviour of Solutions of a Stefan Problem. J. Mathematical Analysis and Applications, 51:633-642, 1975.

8. A. McNabb. Mean Action Times, Time Lags, and Mean First Passage Times for Some Diffusion Problems. Math. Comput. Modelling, 18:123-129, 1993.

9. A. McNabb and R.S. Anderssen. The Freezing Time for Pseudo-Ellipsoids. The Proceedings of The Centre for Mathematics and its Applications Mini-Conference on Free and Moving Boundary and Diffusion Problems, (Editors R.S. Anderssen, J.M. Hill and A.K. Pani, Canberra, June 1990) Australian National University, 30:184-195, 1992.

10. A. McNabb and L. Bass. A Diffusion-Reaction Model for the Cellular Uptake of ProteinBound Ligands. SIAM J. Appl. Math, 51:124-149, 1991.

11. A. McNabb and G. Keady. Diffusion and the Torsion Parameter. J. Austral. Math. Soc. Ser. B, 35:289-301, 1994.

12. A. McNabb and G. C. Wake. Heat Conduction and Finite Measures for Transition Times Between Steady States. IMA Journal of Applied Mathematics, 47:193-206, 1991.

13. B.A. Moffat, K.A. Landman, R.J.W. Truscott, Sweeney, M.H.J. and J.M. Pope. Age related changes in the kinetics of water transport in human eye lenses. Experimental Eye Research, 69:663-669, 1999.

14. J.R. Philip. Hydrostatics and hydrodynamics in swelling soils. Water Resources Research, 5 No. 5:1070-1077, 1969.

15. D.E. Smiles, and M.J. Rosenthal. The movement of water in swelling materials. Aust. J. Soil Res., 6:237-248, 1968.

16. Stapley, A.G.F., Phd Thesis. University of Cambridge, England, 1995. 
17. A.M. Syarief, R.J. Gustafson and R.V. Morey. Moisture diffusion coefficients for yellow-dent corn components. Trans. American Soc. of Agricultural Engineers, 30: 522-528, 1987. 


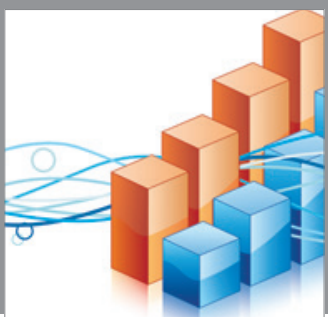

Advances in

Operations Research

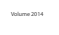

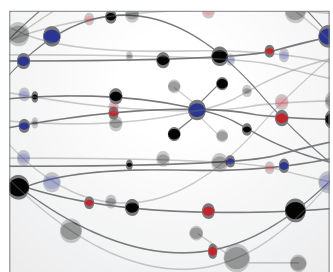

\section{The Scientific} World Journal
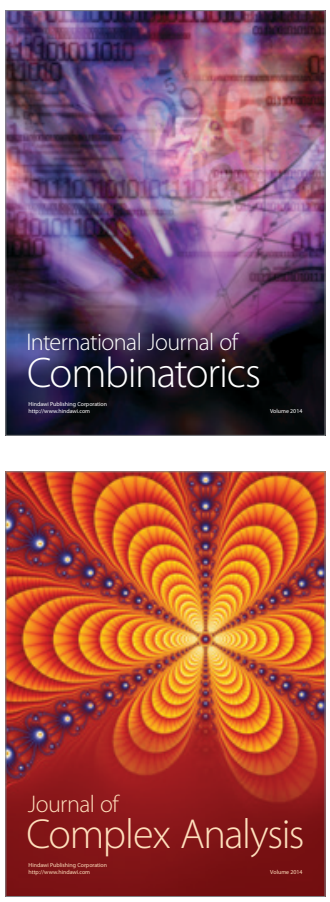

International Journal of

Mathematics and

Mathematical

Sciences
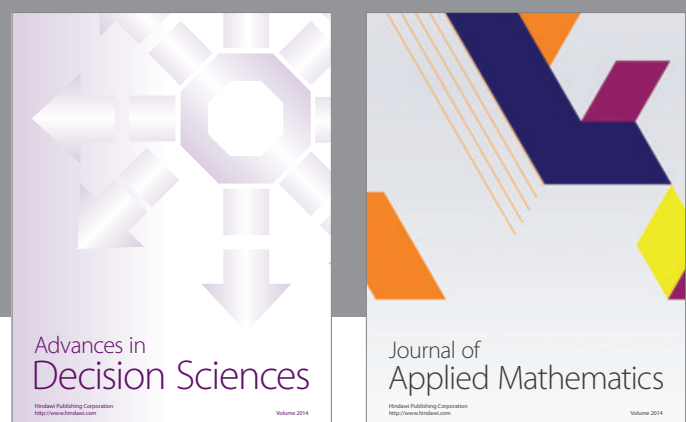

Journal of

Applied Mathematics
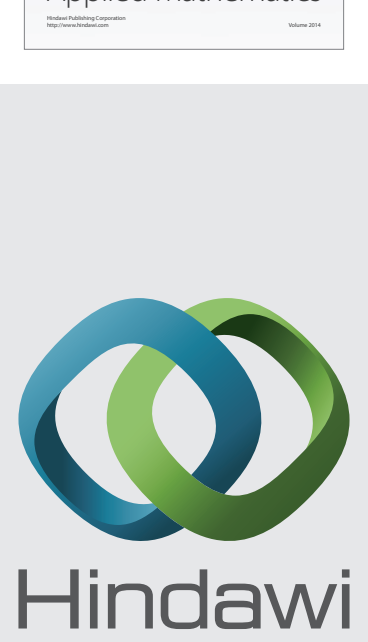

Submit your manuscripts at http://www.hindawi.com
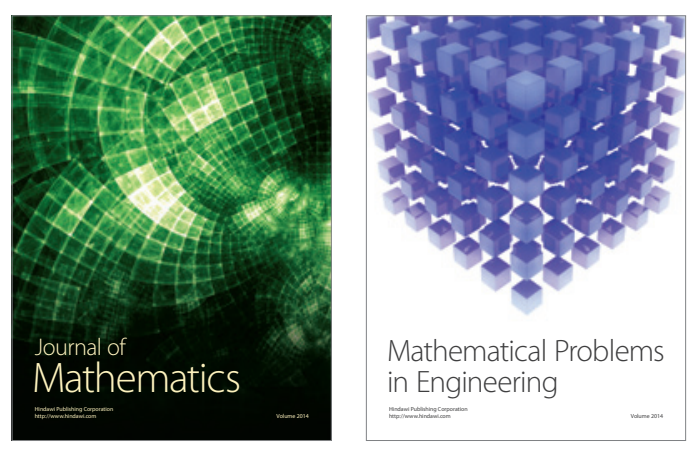

Mathematical Problems in Engineering
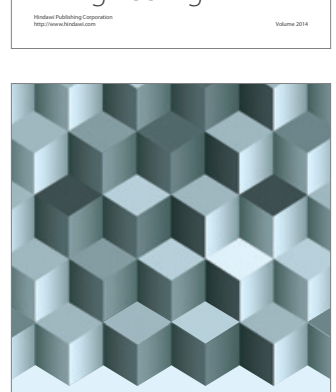

Journal of

Function Spaces
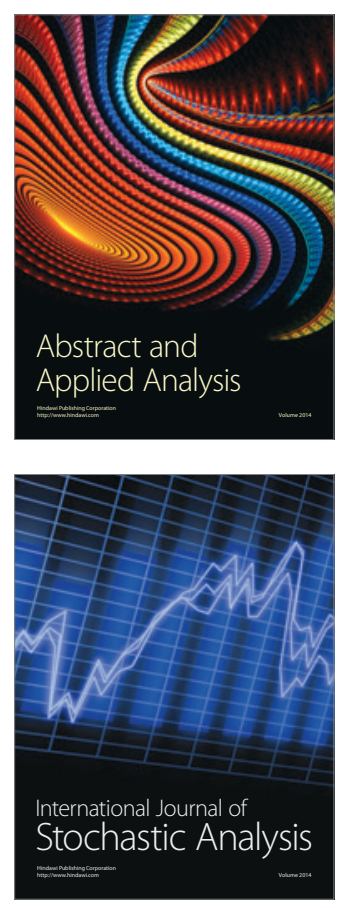

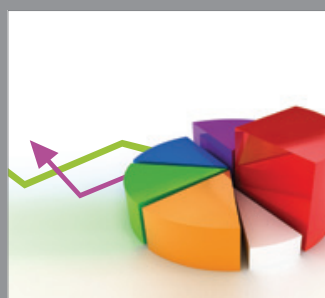

ournal of

Probability and Statistics

Promensencen
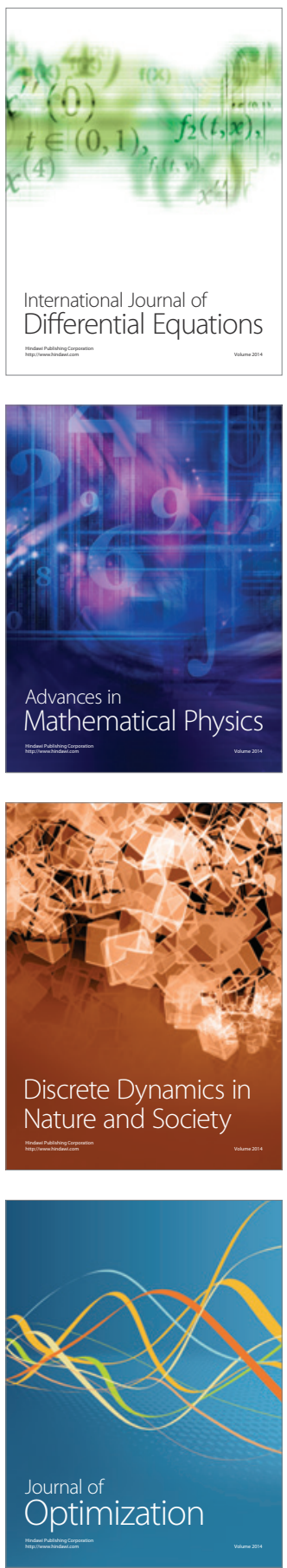